\title{
Linearly-configured BOCDA system with large modulation amplitude using dual-probe wave
}

\author{
Jon Mariñelarena, Yong Hyun Kim, Alayn Loayssa, Kwang Yong Song \\ Department of Electrical and Electronic Engineering, Universidad Publica de Navarra, 31006 Pamplona, Spain \\ Department of Physics, Chung-Ang University, Seoul 156-756, South Korea \\ songky@cau.ac.kr.
}

\begin{abstract}
Brillouin optical correlation domain analysis (LC-BOCDA) system that allows large modulation amplitude $(\Delta f)$ based on a dual probe wave and the control of correlation-order. The adoption of dual probe and the control of correlation-order can suppress the effects of two main origins of the noise the Brillouin loss of the pump and the beat noise between the pump and probe waves. We experimentally confirm that $\Delta f$ and the number of resolving points, $\mathrm{N}$, can be increase to 1.8 times without significant noise.

OCIS codes: $290.5900,060.2370$.
\end{abstract}

\section{Introduction}

Distributed fiber sensors based on stimulated Brillouin scattering (SBS) has become an efficient tool for structural health monitoring thanks to their capability of using the entire optical fiber as a sensor $[1,2]$. One of the most studied sensor techniques is the Brillouin optical correlation domain analysis (BOCDA) which allows distributed measurements with high spatial resolution $(\sim 2 \mathrm{~mm})$ and high sampling rate $(\sim 5 \mathrm{kHz})$ with random access to the sensing position [3].

The linearly-configured BOCDA (LC-BOCDA) scheme has been recently presented and permits a single end access to the fiber under test (FUT), reducing the complexity of the deployment of the FUT [4]. Moreover, the LC-BOCDA has been combined with the differential measurement scheme allowing to double the previous sensing range limit of LC-BOCDA sensors and improving the spatial resolution by more than four folds [5]. However, one unsolved problem of the LC-BOCDA system is the limitation in the amplitude of frequency modulation $(\Delta f)$ which determines the spatial resolution $(\Delta \mathrm{z})$ and the number of sensing points (or resolving points), $N$. In order to improve the performance of the BOCDA sensors it is necessary to increase $\Delta f$, however, in LC-BOCDA systems the maximum value of $\Delta f$ has been practically limited to half of the Brillouin frequency $\left(v_{B} / 2\right)$ of the FUT, because when probe and pump waves overlap, the reflected pump wave cannot be filtered out, and hence, it is added to the detected probe wave, resulting in a significant noise and distortion.

In this paper, we experimentally demonstrate a new measurement configuration for the LC-BOCDA system which allows to double $\Delta f$. It is based on a dual probe wave and correlation-order control that solves the detrimental effect generated by the reflected pump wave and the beat noise due to the spectral overlap between the pump and probe waves.

\section{Fundamentals}

Fig. 1(a) depicts the basics of LC-BOCDA systems, where the probe and pump waves are injected together to the fiber from one end of the FUT. and propagated along the fiber. Both waves are propagated along the fiber and reflected at the far end by a Fresnel reflection using a cleaved end of the fiber [5]. Then, the reflected probe wave counter-propagates to the outgoing pump wave generating strong SBS gain on the probe wave at a correlation peak (CP1) where the frequency offset, $v$, between probe and pump waves is constant. The low reflection at the far end of the fiber suppresses the amplitude of the incoming waves and the SBS gain at CP2, avoiding to have two CP's in the FUT and maintaining the sensing range equal to the distance between CP's.

However, the LC-BOCDA system suffers serious errors when the pump and probe spectra overlap with $\Delta f>v_{B} / 2$. This is because the pump wave cannot be completely filtered out and it is detected together with the probe wave, so, the incoming pump wave experiences strong SBS loss at CP2 when it counter-propagates the high-power outgoing probe wave. The SBS gain and loss signals from the two CP's interfere with each other leading to ambiguous detection and measurement errors.

To overcome these drawbacks, we propose to deploy a dual probe wave in the LC-BOCDA system similarly to the dual probe configuration that has been already adopted in BOTDA systems to reduce the non-local effects [7]. Notice that the Brillouin loss of the pump wave at CP2 is compensated when the pump wave interacts with the upper-sideband of the dual probe wave, obtaining a compensation of the pump loss and allowing to extend $\Delta f$ beyond $v_{\mathrm{B}} / 2$, and, thus, leads to the enhancement of the spatial resolution and the number of resolving points of LC-BOCDA systems. 


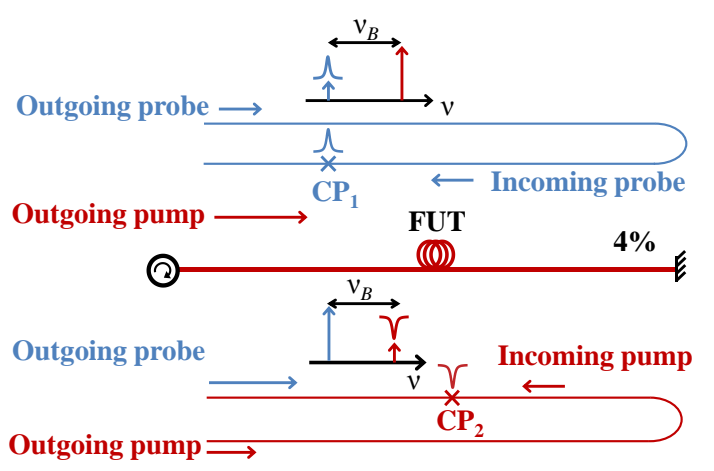

(a)

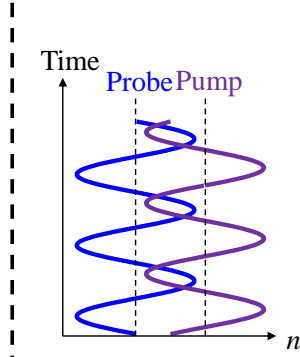

(b.1)

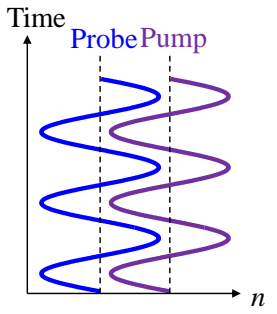

(b.2)

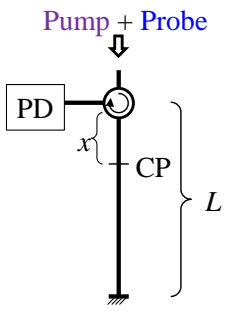

(b.3)

(b)

Fig. 1. (a) Fundamentals of the SBS in LC-BOCDA systems. (b) Brillouin interaction in the dual probe wave scheme.

Another inconvenient generating probe and pump waves with overlapped frequencies in LC-BOCDA systems is that both waves reach simultaneously the PD after its propagation along the FUT and the beat frequency can lie within the bandwidth of PD as schematically shown in Fig.1 (b.1). Since the Brillouin gain is small in the BOCDA system, this beating signal causes a significant noise to the obtained BGS even though the lock-in detection is applied. The occurrence of this beat noise is dependent on the relative phase of the pump and probe modulation waves at the PD, so one can avoid it if the modulation of both waves are controlled 'inphase' as depicted in Fig. 1(b.2). If we consider the situation as shown in Fig. 1(b.3) where the sensing position (i.e. $\mathrm{CP}$ ) is located at $x$ with the length of the fiber $L$, the phase difference, $\Delta f$, at $\mathrm{CP}$ between the outgoing pump and incoming probe is always zero in the BOCDA measurement (by the definition of CP). Therefore $\Delta f$ between the incoming pump and probe waves (that is $\Delta f$ at the PD) is determined by the roundtrip path length, $2 n(L-x)$, which should be close to $2 \pi$ in order to suppress the beat noise.

\section{Experimental setup and results}

Fig. 2 depicts the experimental setup of the LC-BOCDA system that was deployed to demonstrate the proposed configuration. A 1550-nm laser diode (LD) was used as a light source and a sinusoidal RF signal was applied to modulate the optical source. The output signal of the LD was divided into two branches using a 3-dB coupler. One of them was used to generate the single-sideband probe wave of a conventional BOCDA system using a single-sideband modulator (SSBM). In the case of generating a dual probe wave to compare the performance of the proposed technique and the conventional setup, a Mach-Zehnder electro-optic modulator (MZ-EOM) was used to create two sidebands.

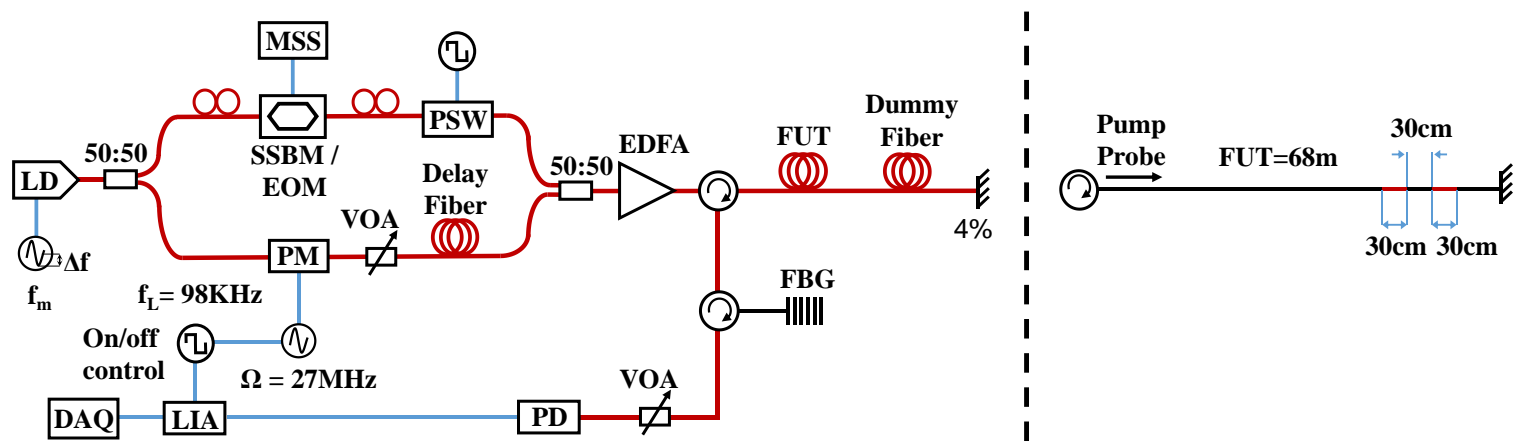

Fig. 2. Experimental setup of the LC-BOCDA system using the differential measurement scheme. The inset shows the structure of the $68-\mathrm{m}$ FUT where two test sections of $30 \mathrm{~cm}$ were deployed: VOA, variable optical attenuator, PD, photo-detector, LIA, lock-in amplifier.

In both configurations, the SSBM and the MZ-EOM were driven by a microwave sweep synthesizer (MSS) that scans $\Delta v$ for each position of the fiber. A polarization switch (PSW) was employed on the probe wave branch for suppressing the polarization dependence before injecting the probe wave into the fiber. In the lower branch, a phase-modulation at a fixed frequency was applied to the pump wave to use the differential measurement scheme [6]. Then, the pump wave was launched to a 5-km delay SMF to use a high-order CP. Both signals, probe and pump waves were amplified by a high output power $(27 \mathrm{dBm})$ Erbium-doped fiber amplifier (EDFA) and launched to a 68-m SMF FUT. The far end of the FUT was cleaved to weakly reflect the pump and probe waves. A fiber Bragg grating (FBG) was used to suppress the ASE noise and reduce the pump wave component before the detection. Fig. 3 shows the optical spectra and relative wavelength position of the 
optical filter, the dual-probe wave and the pump wave before and after de filter. The probe-wave was filtered and the higher sideband was completely suppressed, so the contribution of the second sideband was negligible in the detection. However, the pump wave could not be completely filtered out and the overlapped frequencies are detected at the photo-detector (PD) and added to the probe wave signal, adding the additional SBS interaction when single-sideband probe wave configuration is deployed.

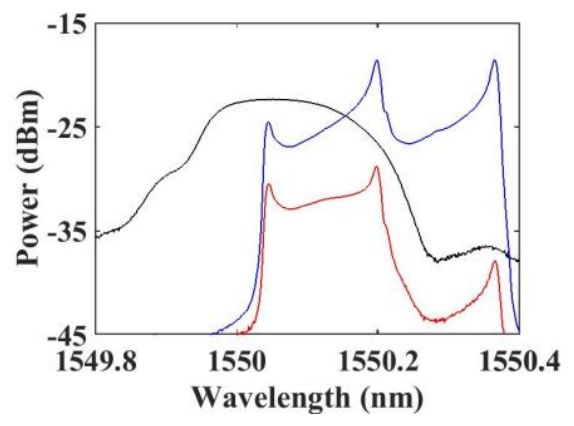

(a)

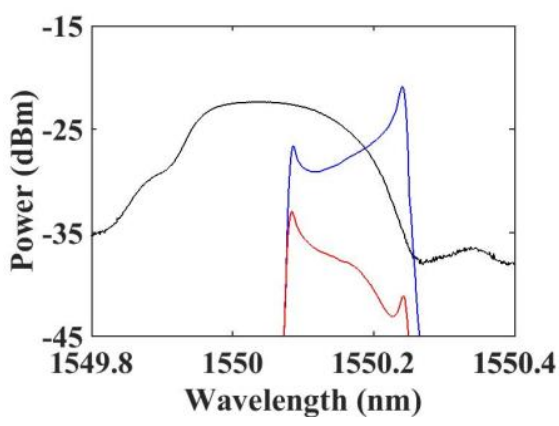

(b)

Fig. 3. Optical spectra of the double-sideband modulation (a) and pump wave (b) before (blue line) and after (red line) filtering using frequency deviation of $9 \mathrm{GHz}$.

For the acquisition of the Brillouin gain spectrum (BGS) at every position of the fiber, lock-in detection and differential measurement technique were applied to reconstruct the spectrum which is based on detecting the difference probe wave signal between the on and off of the phase-modulated pump wave. Two strain sections of $30-\mathrm{cm}$ length with separation of 30-cm fiber were deployed near the end of the FUT as shown in the inset of Fig. 2. We connected a dummy fiber $(\sim 133 \mathrm{~m})$ to the end of the FUT as shown in Fig. 2 and adjusted the order of $\mathrm{CP}$ to avoid the beat noise. The sensing position was scanned with a $15 \mathrm{~cm}$ step to perform distributed measurements by changing simultaneously $f_{m}$ and the $\mathrm{CP}$ order.

Fig. 4 (a) and (b) show the normalized BGS measured at one of the strain sections for different $\Delta f$ 's $(5,7$ and $9 \mathrm{GHz}$ ) using the single or dual probe wave configuration. In Fig. 4(a), two main peaks were simultaneously observed in the BGS when the single-sideband probe wave was deployed, which are due to the contribution of Brillouin loss spectrum (BLS) of the pump wave measured at another CP position as explained in Fig. 1(a). In particular, the peak at higher frequency in Fig. 4(a) is from the CP position where the strain was applied, which is generated by the interaction between the outgoing pump and the incoming probe wave. The second BGS that appears at lower frequency, that we call ghost peak, is the result of the interaction between the incoming pump and the outgoing probe at another position of the FUT. The ghost peak is caused by the depletion that the high power probe wave generates on the counter-propagating pump wave. As shown, the amplitude of the ghost peak is dependent on the overlap, confirming that this configuration is intolerant to the probe and pump overlap. Note that the resultant ghost peak instead of showing a negative Brillouin loss spectrum as explained previously, it has a similar shape to that of the BGS detected in the strain section position. This is possible because the lock-in amplifier was operated in an amplitude mode (or R-mode). When an in-phase mode (or X-mode) is used in the lock-in detection the sign of the ghost peak fluctuates according to the sensing position. Observe also that there are side lobes apart from the two BGS, which are due to the intrinsic shape of the BGS when differential measurement technique is used with the module operation mode of the LIA [6]. The amplitude of the side lobes are always smaller than the main peaks, therefore, there are no detrimental effects of the distortion.

Notice that even when there was no overlap between probe and pump using $\Delta f \sim 5 \mathrm{GHz}$, a small ghost peak was detected simultaneously to the real BGS due to the non-ideal filtering of the pump wave. The pump wave was attenuated but not completely suppressed, so a remaining pump wave was detected and added to the probe wave. Regarding the amplitude on the BGS, it is well-known that its amplitude depends on the spatial resolution $\Delta z$, so, all the measured BGS in Fig. 4(a) and (b) were normalized to the amplitude of the BGS peak in the strain section when $\Delta f$ is $9 \mathrm{GHz}$ for comparison.

Fig. 4(b) depicts the BGS measured under the same conditions but using the dual probe wave configuration. On the contrary to Fig. 4(a), it allows to retrieve the correct BGS without detecting the ghost peak for different values of $\Delta f$. As explained before, using the dual probe wave scheme, the pump wave suffers gain and loss simultaneously due to the interaction with the two sidebands, so that, the net gain of the pump is null. Therefore, it is experimentally demonstrated that $\Delta f$ can be increased over $5 \mathrm{GHz}$ without distorting the measured BGS. The modulation frequencies of the LD that were used for distributed measurements were in the range from 0.69 to $1.09 \mathrm{MHz}$. The nominal spatial resolution was around $27 \mathrm{~cm}$ for $\Delta f=5 \mathrm{GHz}$ and $15 \mathrm{~cm}$ for $\Delta f=9 \mathrm{GHz}$ when the measurement range $d_{m}$ is equal to $98 \mathrm{~m}$.

In order to experimentally demonstrate a correct performance of the proposed technique when large frequency deviation of LD is used, we performed distributed measurements of $v_{\mathrm{B}}$ along the FUT using both configurations with different $\Delta f$ 's. Fig. 4(c) shows the resultant map of $v_{\mathrm{B}}$ for the conventional scheme with the 
single probe wave where it is observed that there are multiple errors on the calculation only when there is overlap of pump and probe. These errors are due to the negative effects of the ghost BGS, which distorts the real BGS as explained previously. Meanwhile, Fig. 4(d) highlights that using the dual probe wave scheme the $v_{B}$ is accurately obtained without errors for any $\Delta f$ deployed, confirming that the proposed technique permits to extend $\Delta f$ over the current limit. A zoomed view of the $v_{\mathrm{B}}$ distribution near the strain sections in the FUT is shown in Fig. 4(d), confirming that the applied strain is correctly measured even when $\Delta f$ of $9 \mathrm{GHz}$ is used. A variation of $197 \mathrm{MHz}$ is observed with $\Delta f$ of $9 \mathrm{GHz}$ and $203 \mathrm{MHz}$ with $5 \mathrm{GHz}$ and $7 \mathrm{GHz}$, which matches well with the theoretical value for the applied strain of $4000 \mu \varepsilon$ considering the slope of $\sim 0.05 \mathrm{MHz} / \mu \varepsilon$.

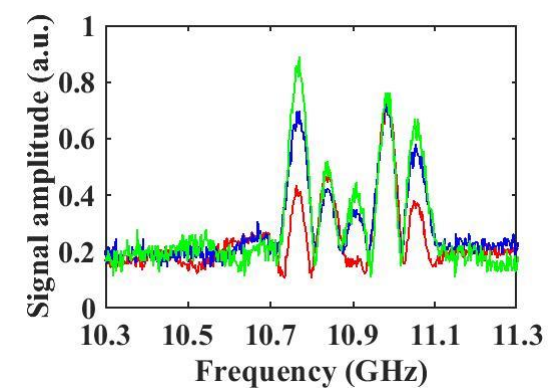

(a)

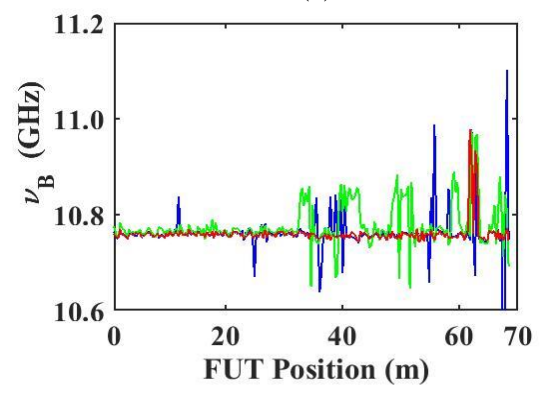

(c)

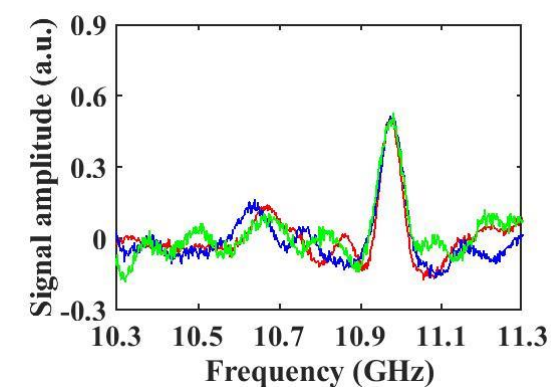

(b)

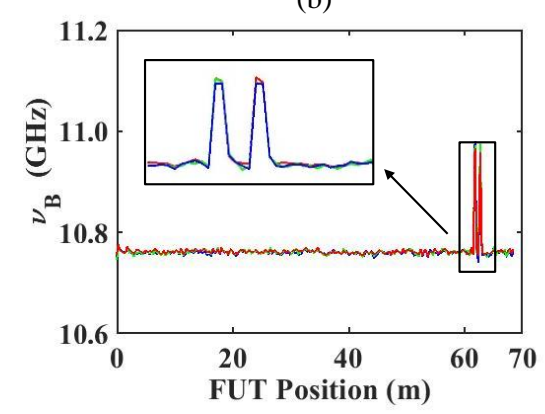

(d)

Fig. 3. Measured $v_{B}$ of the FUT using (a) single-probe wave and (b) dual-probe wave configuration with zoomed view near the strain-applied section for different $\Delta f$ 's ( $5 \mathrm{GHz}$ red, $7 \mathrm{GHz}$ blue, $9 \mathrm{GHz}$ green).

\section{Conclusions}

In summary, we have introduced a new measurement configuration to a linearly configured BOCDA system which allows enlarged modulation amplitude using the dual probe wave and the control of correlation-order. We experimentally confirmed that the proposed technique permits to increase the modulation amplitude from the former limit of $5 \mathrm{GHz}$ to $9 \mathrm{GHz}$ which corresponds to the enhancement of nearly twice the spatial resolution or the number of resolving points in the BOCDA system.

\section{Acknowledgments}

The authors wish to acknowledge the support from the Spanish Ministerio de Economia y Competitividad through the project TEC2016-76021-C2-1-R, FEDER funds and Universidad Publica de Navarra.

\section{References}

[1] T. Horiguchi and M. Tateda, "BOTDA-nondestructive measurement of single-mode optical fiber attenuation characteristics using Brillouin interaction: theory," J. Lightw. Technol. 7, 1170-1176 (1989).

[2] X. Bao et al., “32-km distributed temperature sensor based on Brillouin loss in an optical fiber,” Opt. Lett. 18, 1561-1563 (1993).

[3] K. Hotate and T. Hasegawa, "Measurement of Brillouin Gain Spectrum Distribution along an Optical Fiber Using a Correlation-Based Technique - Proposal, Experiment and Simulation,” IEICE Trans. Electron. E83-C, 405-412 (2000).

[4] K. Y. Song and K. Hotate, "Brillouin Optical Correlation Domain Analysis in Linear Configuration" IEEE Photon. Technol. Lett. 20, 2150-2152 (2008).

[5] J. H. Jeong et al., "Linearly configured BOCDA system using a differential measurement scheme,” Opt. Express 22, 1467-1473 (2014).

[6] J. H. Jeong et al., "Differential measurement scheme for Brillouin Optical Correlation Domain Analysis,” Opt. Express 20, 27094-27101 (2012).

[7] A. Minardo and R. Bernini and L. Zeni, “A Simple Technique for Reducing Pump Depletion in Long-Range Distributed Brillouin Fiber Sensors," IEEE Sensors Journal 9, 633-634 (2009). 